\title{
Measurements of the Size and Pressure of the Local Bubble Towards Eridanus
}

\author{
D.N. Burrows ${ }^{1}$ and Z. Guo ${ }^{2}$
}

1 Department of Astronomy \& Astrophysics, Pennsylvania State University, 525 Davey Lab, University Park, PA 16802, USA

2 Goddard Space Flight Center, Code 631, Greenbelt, MD 20771, USA

\begin{abstract}
We have measured the size and pressure of the Local Bubble in the direction of the constellation Eridanus using ROSAT PSPC observations of soft $\mathrm{X}$-ray "shadows" cast by neutral clouds against the bright X-ray emission from the Orion-Eridanus superbubble. We discuss the technique and present our results for the size of the Local Bubble in the directions of our shadowing fields. Using these distances and the measured soft $\mathrm{X}$-ray emission measures, we derive the Local Bubble's thermal pressure. For four lines of sight in Eridanus, we obtain pressures of about $1.2 \times 10^{4} \mathrm{~K} \mathrm{~cm}^{-3}$, in good agreement with measurements in several other directions.
\end{abstract}

\section{Introduction}

Since the discovery of soft X-ray shadows in Draco (Snowden et al. 1991, Burrows \& Mendenhall 1991), X-ray shadows observed by the ROSAT PSPC have been used to measure the brightness of the galactic halo, investigate cloud properties, study the Local Bubble, and set limits on the extragalactic diffuse background. Over the past several years, we have used ROSAT AllSky Survey data and pointed observations to study the X-ray emission from the Orion-Eridanus superbubble (Snowden et al. 1995, Guo et al. 1995, Guo et al. 1997). Here, we summarize our results on the size and pressure of the Local Bubble in this direction.

\section{Technique}

This work is based on a multiwavelength investigation of the Orion-Eridanus superbubble, incorporating data in four wavelengths:

1. IR: IRAS $100 \mu \mathrm{m}$ maps of the Orion-Eridanus superbubble trace the cold cirrus clouds with an angular resolution of about $5^{\prime}$.

2. X-ray: ROSAT PSPC observations (both scanning and pointed) were compared with the IR maps to find soft X-ray shadows. The X-ray pointed observations also provided multicomponent spectral modeling which gave 
the emission measure of the unabsorbed component produced in the Local Bubble.

3. $21 \mathrm{~cm}: \mathrm{N}_{\mathrm{H}}$ maps of ROSAT pointed fields were made using the 140 foot Green Bank telescope of NRAO. We scanned through the velocity channel maps to find velocities corresponding to the IR/X-ray shadow morphology.

4. Optical: Na D absorption line spectroscopy towards stars in these fields allowed us to place limits on the distance to the absorbing clouds, based on the presence or absence of optical absorption features at the velocities found from the $\mathrm{N}_{\mathrm{H}}$ maps.

The outcome of this analysis is a series of distance estimates to the absorbing clouds. By studying these absorbing features over a large solid angle, and by comparison with $\mathrm{H} \alpha$ measurements (Reynolds \& Ogden 1979), we have concluded that the pervasive velocity component observed in $21 \mathrm{~cm}$ emission, $\mathrm{H} \alpha$ emission, and $\mathrm{Na} \mathrm{D}$ absorption in the LSR range +5 to $+10 \mathrm{~km} / \mathrm{s}$ is probably produced by material in the shell of the Local Bubble, while the components observed in the -20 to $-30 \mathrm{~km} / \mathrm{s}$ range originate in the near shell of the Orion-Eridanus superbubble (Guo et al. 1995, Guo et al. 1997).

We note evidence for interaction between the two shells in a field at $(\ell, b)=$ $\left(200^{\circ},-47^{\circ}\right)$, where both velocity components appear in the optical data over a narrow distance interval (Guo et al. 1995). Further evidence supporting an interaction between these bubbles comes from a $21 \mathrm{~cm}$ cloud in this field which has identical morphology in both the +4 and $-25 \mathrm{~km} / \mathrm{s}$ channels, suggesting that it is being crushed between the shells (Guo et al. 1995).

\section{Results}

In our previous paper (Guo et al. 1995), we showed that the field at $(\ell, b)=$ $\left(200^{\circ},-47^{\circ}\right)$ has a well-determined distance of about $159 \mathrm{pc}$ for both the Local Bubble shell and the Orion-Eridanus shell. The local emission measure determined from the spectral fit for this field was $0.0057 \mathrm{~cm}^{-6} \mathrm{pc}$, using a solar abundance CIE plasma code (Raymond \& Smith 1977) with a temperature of $10^{6} \mathrm{~K}$. The pressure inferred for this line of sight is $1.2 \times 10^{4} \mathrm{~cm}^{-3} \mathrm{~K}$, in good agreement with the value found towards MBM12 by Snowden et al. 1993.

In Guo et al. 1997 we extend this result with the addition of three new fields for which we have obtained reasonably well-constrained distance estimates for the Local Bubble shell. These results are summarized in Table 1. They show a trend of decreasing shell distance with increasing latitude, but the derived pressure is quite uniform with a mean value of about $1.2 \times 10^{4}$ $\mathrm{cm}^{-3} \mathrm{~K}$. For comparison, we include two measurements of the Local Bubble pressure by other authors using a similar technique. 
Table 1. Local Bubble Density \& Pressure

\begin{tabular}{ccccc}
\hline$(\ell, b)$ & $\begin{array}{c}\text { X-ray EM } \\
\left(\mathrm{cm}^{-6} \mathrm{pc}\right)\end{array}$ & $\begin{array}{c}\mathrm{d} \\
(\mathrm{pc})\end{array}$ & $\begin{array}{c}\mathrm{N}_{\mathrm{e}} \\
\left(\mathrm{cm}^{-3}\right)\end{array}$ & $\begin{array}{c}P / k \\
\left(\mathrm{~cm}^{-3} \mathrm{~K}\right)\end{array}$ \\
\hline$\left(190^{\circ},-48^{\circ}\right)$ & 0.0050 & 177 & 0.0053 & $1.1 \times 10^{4}$ \\
$\left(200^{\circ},-47^{\circ}\right)$ & 0.0057 & 159 & 0.0060 & $1.2 \times 10^{4}$ \\
$\left(200^{\circ},-43^{\circ}\right)$ & 0.0045 & 130 & 0.0059 & $1.2 \times 10^{4}$ \\
$\left(206^{\circ},-40^{\circ}\right)$ & 0.0049 & 101 & 0.0070 & $1.4 \times 10^{4}$ \\
\hline$\left(159^{\circ},-34^{\circ}\right)^{\dagger}$ & 0.0024 & 65 & 0.006 & $1.3 \times 10^{4}$ \\
$\left(166^{\circ},-32^{\circ}\right)^{\ddagger}$ & 0.0077 & $<40$ & $>0.014$ & $>1.9 \times 10^{4}$ \\
\hline
\end{tabular}

Snowden et al. 1993

$\ddagger$ Bowyer et al. 1995

\section{Discussion}

The fact that independent measurements towards clouds in different parts of the sky agree on a pressure consistent with that needed to support the weight of the interstellar medium (Cox \& Snowden 1986) is encouraging, but should be considered in light of two important caveats.

First, the pressures reported here are model-dependent. Specifically, the emission measures used to obtain the density (and hence, the pressure), assume collisional ionization equilibrium (CIE) conditions in a plasma with solar abundances, a condition known to be unlikely on the basis of DXS spectra of the soft X-ray diffuse background (Sanders et al. 1993). For example, if the gas is depleted but is still in CIE, a higher emission measure is required to produce the observed soft $\mathrm{X}$-ray intensity. The pressure, which scales as the square root of the emission measure, will increase accordingly. Nonequilibrium (NEI) models will also produce different emission measures (and hence different pressures). Even different CIE models produce different results: Berghöfer reported at this meeting that the EUV pressure given in Table 1 for the $\left(166^{\circ},-32^{\circ}\right)$ field, which was based on spectral models by Landini \& Monsignori-Fossi (1990), is in good agreement with the other fields listed in Table 1 if a Raymond \& Smith plasma code is used instead.

The second important caveat concerns the distance estimates. We obtain the plasma density by dividing the emission measure by the path length, assuming uniform emissivity within the Local Bubble. If the emissivity is peaked at the edge of the cavity or in cloud boundaries, then we may be seriously underestimating the thermal pressure. Even if the volume emissivity is uniform, these pressures hinge on our distance estimates to the edge of the Local Bubble, and on the assumption that the boundary of the cavity surrounding the Sun is the same as the boundary of the hot plasma (an assumption we believe is violated in the direction of the "Welsh tunnel", where the cavity extends to great distances without a corresponding increase in the soft X-ray intensity). Finally, the distances we have used are based on stellar distances obtained by spectral parallax, a notoriously unreliable 
technique (but the best available to us at the time). We are in the process of revisiting these results using more reliable Hipparcos distances; the revised distances and pressures will be incorporated into Guo et al. 1997.

\section{References}

Bowyer, S., Lieu, R., Sidher, S. D., Lampton, M., \& Knude, J. 1995, Nature, 375, 212

Burrows, D. N., \& Mendenhall, J. A. 1991, Nature, 351, 629

Cox, D. P.,\& Snowden, S. L. 1986, Adv. Space Res., 6, 97

Guo, Z., Burrows, D. N., Sanders, W. T., Snowden, S. L., \& Penprase, B. E. 1995, ApJ, 453, 256

Guo, Z., Burrows, D. N., Penprase, B. E., \& Sanders, W. T. 1997, ApJ, submitted Landini, M., \& Monsignori-Fossi, G. C. 1990, A\&AS, 82, 229

Raymond, J. C., \& Smith, B. W. 1977, ApJS, 35, 419 (and unpublished updates used in XSPEC code)

Reynolds, R. J., \& Ogden, P. M. 1979, ApJ, 229, 942

Sanders, W. T., et al. 1993, Proc. SPIE: EUV, X-ray and Gamma-Ray Instrumentation in Astronomy IV, 2006, 221

Snowden, S. L., Burrows, D. N., Sanders, W. T., Aschenbach, B., \& Pfeffermann, E. 1995, ApJ, 439, 399

Snowden, S. L., McCammon, D., \& Verter, F. 1993, ApJ, 409, L21

Snowden, S. L., Mebold, U., Hirth, W., Herbstmeier, U., \& Schmitt, J. H. M. M. 1991, Science, 252, 1529 